\title{
On numerical solution of nonlinear parabolic multicomponent diffusion-reaction problems
}

\author{
Gh. Juncu, C. Popa, Gh. Sarbu
}

\begin{abstract}
This work continues our previous analysis concerning the numerical solution of the multi-component mass transfer equations. The present test problems are two-dimensional, parabolic, non-linear, diffusion- reaction equations. An implicit finite difference method was used to discretize the mathematical model equations. The algorithm used to solve the non-linear system resulted for each time step is the modified Picard iteration. The numerical performances of the preconditioned conjugate gradient algorithms (BICGSTAB and GMRES) in solving the linear systems of the modified Picard iteration were analysed in detail. The numerical results obtained show good numerical performances.
\end{abstract}

\section{INTRODUCTION}

The generalized Fick law, shows that, for a multicomponent mixture, the diffusion of a certain species is dictated not only by its own concentration gradient but also by the concentration gradients of the other species. This idea was first formulated by Maxwell [13] and Stefan [16].

From a computational point of view, the numerical solution of multicomponent diffusion equations is still difficult and is considered an open problem. The numerical algorithms used to solve the partial differential equations that

Key Words: Multi-component diffusion, Finite differences, Picard Method, Diffusionreaction equation.

2010 Mathematics Subject Classification: Primary 46G05, 46L05; Secondary 47A30, $47 \mathrm{~B} 47$.

Received: 21.12 .2020

Accepted: 12.04 .2021 
model multicomponent mass transfer are reviewed in $[3,4]$.

Relating to the parabolic partial differential equations case, the subject of the present work, the followings aspects must be mentioned: (1) for 1D spatial operators, the method of lines (discretization of the spatial derivatives with finite difference, finite volume, or Galerkin methods and the integration of the resulting ODE system with an ODE solver, e.g., Livermore Solver for Ordinary Differential Equations (LSODE) package) was used in [12, 18]; (2) discretization of the spatial derivatives with the finite difference, finite volume, or Galerkin methods and time integration with the implicit (Euler), implicitexplicit (Euler), or Crank-Nicholson algorithm are the numerical methods employed in $[8,9,14,17,20]$ for $2 \mathrm{D}$ spatial operators.

Kumar and Mazumder [8] have developed a numerical solution algorithm for multicomponent diffusion equations. The first step of the method was to decompose the domain of definition into subdomains, using the binary spatial partitioning algorithm. For each subdomain the equations of the mathematical model were discretized using the finite volume method. Discrete linear equations were solved, for each subdomain, with the preconditioned GMRES algorithm.

Kozeschnik [9] use the finite-element technique, to discretize the multicomponent diffusion equations. The suggested formalism is based on the solution of the integral statement of the generalized diffusion equation. This treatment allows for a simple implementation of particular boundary conditions and can easily be extended from a one to a multidimensional analysis. The finite-element diffusivity matrices are evaluated for a one-dimensional bar and a two-dimensional triangular element.

Wangard et al. [20] approaches the solving of the equations governing laminardiffusion of multicomponent fluids (gas or liquid). The rigorous model for multicomponent mass diffusion is recast into a mathematical form analogous to that for binary diffusion. Diagonal terms are integrated by default and terms outside the diagonal are explicitly integrated.

Mazumder [14] analyzes the case of two-dimensional diffusion, and draws the following conclusions: (1) if only the self-diffusion operator is treated implicitly, the stability of the resulting equations depends on the constraint conditions imposed on the total conservation of mass; (2) a totally implicit procedure prevents this instability and ensures an unconditional stability.

Bottcher [17] analyzes a multicomponent stationary transport problem, which combines diffusion with fluid flow. The discretization of the mathematical model was performed with the finite element method. Solving the resulting nonlinear algebraic system was performed using the Newton-Raphson method. Juncu [5] and Juncu et al. [6,7] develop a new splitting method for solving the multicomponent diffusion problem, the linear case. The method is stable 
if the discrete spatial operator is symmetric and positive definite.

The objective of the present work is the analysis of the numerical performances of the modified Picard-preconditioned conjugate gradient algorithms (BICGSTAB and GMRES) for solving 2D, parabolic, non-linear multicomponent diffusion-reaction equations. It must be mentioned that these algorithms were used in [3] for solving 2D elliptic, non-linear, multicomponent diffusionreaction equations.

The present paper is organized as follows: in Section 2 we describe the mathematical model of the test problems. Section 3 presents the numerical algorithms. The results of the numerical simulations performed are presented in Section 4. Finally, some concluding remarks are briefly mentioned in Section 5 .

\section{THE DIFFUSION-REACTION PROBLEM}

Consider that, inside a homogeneous porous catalyst pellet having the finite slab geometry with square section, the isothermal, first-order, complex chemical reaction,

$$
\begin{gathered}
A_{1} \quad \stackrel{k_{1}^{*}}{\longrightarrow} A_{2} \underset{k_{4}^{*}}{\stackrel{k_{2}^{*}}{\rightleftarrows}} A_{3} \\
A_{1} \underset{k_{5}^{*}}{\stackrel{k_{3}^{*}}{\rightleftarrows}} A_{4}
\end{gathered}
$$

takes place. The external transport resistances are assumed negligibly (the concentrations on the external surface of the catalyst pellet are the same as the bulk values). When the total mixture molar concentration is constant, the dimensionless concentrations profiles inside the pellet are given by

$$
\frac{\partial Z_{i}}{\partial t}=\sum_{j=1}^{4}\left(\frac{\partial}{\partial x} D_{i, j} \frac{\partial Z_{j}}{\partial x}+\frac{\partial}{\partial y} D_{i, j} \frac{\partial Z_{j}}{\partial y}\right)+R_{i}, \quad i=\overline{1,4}
$$

where

$$
\begin{gathered}
R_{1}=-k_{1} Z_{1}-k_{3} Z_{1}+k_{5} Z_{4} \\
R_{2}=k_{1} Z_{1}-k_{2} Z_{2}+k_{4} Z_{3} \\
R_{3}=k_{2} Z_{2}-k_{4} Z_{3}
\end{gathered}
$$




$$
R_{4}=k_{3} Z_{1}-k_{5} Z_{4}
$$

where $Z_{i}=A_{i} / A_{1 b}$ is the dimensionless concentration, $A_{1 b}$ is the bulk molar concentration of species $A_{1}, k_{i}=k_{i}^{*} L^{2} / D_{\text {ref }}$ is the nondimensional reaction rate constant, $L$ is the length of the square section, $t=t D_{r e f} / L^{2}$ is the dimensionless time or Fourier number, $D_{i, j}$ is the multicomponent Fick diffusion coefficient related to $D_{r e f}$, and $D_{r e f}$ is a reference diffusion coefficient. In our case (diffusion-reaction systems), we can use the molar average frame.

The initial and boundary conditions for the mathematical model previously presented are :

-initial conditions :

$$
t=0, \quad Z_{1}=0, \quad Z_{2}=Z_{3}=Z_{4}=0 \quad(x, y) \in \Omega=(0,1) \times(0,1)
$$

-boundary conditions:

$$
t \in(0,1), \quad Z_{1}=1, \quad Z_{2}=Z_{3}=Z_{4}=0 \quad(x, y) \in \partial \Omega
$$

The relation used to compute the matrix of the mole-based multicomponent Fick diffusion coefficients, $D$, is,

$$
D=B^{-1} \Gamma
$$

In relation (5), $B$ is the matrix that takes into account the drag effects and $\Gamma$ is the matrix of thermodynamic effects. Considering negligible the Knudsen and surface diffusion, the matrix $B$ is given by, $[1,10,19]$,

$$
\begin{gathered}
B_{i i}=\frac{x_{i}}{\mathrm{D}_{i n}}+\sum_{\substack{k=1 \\
k \neq i}}^{n} \frac{x_{k}}{\mathrm{D}_{i k}}, i=1, \ldots, n-1, \\
B_{i j}=-x_{i}\left(\frac{1}{\mathrm{D}_{i j}}-\frac{1}{\mathrm{D}_{i n}}\right), i, j=1, \ldots, n-1, i \neq j,
\end{gathered}
$$

where $\bigoplus_{i j}$ is the Stefan-Maxwell diffusion coefficient related to $D_{r e f}$ and $x_{i}$ is mole fraction of species $i$. The mole fraction of the $i$-th species, $x_{i}$, is calculated by:

$$
x_{i}=\frac{Z_{i}}{\sum_{j=1}^{n} Z_{j}}
$$

where $n$ is the total number of chemical species present in system. In this work the value assumed for $n$ is $n=5$. The fifth species is an inert not involved in the 
chemical reaction. The dimensionless molar concentration of $Z_{5}$ is computed based on the assumption of constant total mixture molar concentration. In addition, it must be mentioned that for a mixture with $\mathrm{n}$ chemical species, there are only n-1 linear independent fluxes and composition gradients, [19]. For this reason, only n-1 mass balance equations are necessary to model such a system. The Stefan-Maxwell diffusion coefficients were considered constants. This assumption is a widely used one, [19]. The reference diffusion coefficient $D_{\text {ref }}$ selected is $D_{\text {ref }}=\mathrm{B}_{15}$

\section{NUMERICAL METHODS}

\subsection{Discretization}

The spatial derivatives of Eq. (2) were discretized with the central secondorder accurate finite difference scheme on uniform grids with $N \times N$ points:

$$
\begin{gathered}
0=x_{1}<x_{2} \ldots<x_{N-1}<x_{N}=1, \quad x_{k}=(k-1) h, \\
0=y_{1}<y_{2} \ldots<y_{N-1}<y_{N}=1, \quad y_{l}=(l-1) h, \quad k, l=\overline{0, N}
\end{gathered}
$$

where $h=1 /(N-1)$ is the grid step size. The discrete approximation obtained is:

$$
\begin{array}{r}
\frac{Z_{i}^{k, l, t+\Delta t}-Z_{i}^{k, l, t}}{\Delta t}=\sum_{j=1}^{4}\left[\frac{D_{i, j}^{k+\frac{1}{2}, l}\left(Z_{j}^{k+1, l, t+\Delta t}-Z_{j}^{k, l, t+\Delta t}\right)}{h^{2}}-\right. \\
-\frac{D_{i, j}^{k-\frac{1}{2}, l}\left(Z_{j}^{k, l, t+\Delta t}-Z_{j}^{k-1, l, t+\Delta t}\right)}{h^{2}}+ \\
\quad+\frac{D_{i, j}^{k, l+\frac{1}{2}}\left(Z_{j}^{k, l+1, t+\Delta t}-Z_{j}^{k, l, t+\Delta t}\right)}{h^{2}}- \\
\left.-\frac{D_{i, j}^{k, l-\frac{1}{2}}\left(Z_{j}^{k, l, t+\Delta t}-Z_{j}^{k, l-1, t+\Delta t}\right)}{h^{2}}\right]+R_{i}
\end{array}
$$

where $i=\overline{1,4}$.

The values of the diffusion coefficients were calculated as:

$$
\begin{array}{ll}
D_{i, j}^{k+\frac{1}{2}, l}=\frac{D_{i, j}^{k+1, l}+D_{i, j}^{k, l}}{2}, & D_{i, j}^{k-\frac{1}{2}, l}=\frac{D_{i, j}^{k, l}+D_{i, j}^{k-1, l}}{2} \\
D_{i, j}^{k, l+\frac{1}{2}}=\frac{D_{i, j}^{k, l+1}+D_{i, j}^{k, l}}{2}, & D_{i, j}^{k-\frac{1}{2}, l}=\frac{D_{i, j}^{k, l-1}+D_{i, j}^{k, l}}{2}
\end{array}
$$


The grid point values of the multicomponent Fick diffusion coefficients were calculated with the relation (5) presented in the previous section.

\subsection{Numerical Algorithms}

The nonlinear algorithm used in the present work is the modified Picard iteration [11].

The modified Picard scheme, also called a fixed point method, is given by:

$$
\begin{gathered}
A\left(Z^{m}\right) \delta Z^{m+1}=b\left(Z^{m}\right)-A\left(Z^{m}\right) Z^{m} \\
Z^{m+1}=Z^{m}+\delta Z^{m+1}
\end{gathered}
$$

where $m$ is the iteration number such that $Z^{0}$ is the initial estimate, $Z^{m}=$ $\left[Z_{1}^{m}, Z_{2}^{m}, Z_{3}^{m}, Z_{4}^{m}\right]$. The matrix $A\left(Z^{m}\right)$ is defined by:

$A\left(Z^{m}\right)=\left[\begin{array}{cccc}A_{11}^{m} & A_{12}^{m} & A_{13}^{m} & A_{14}^{m} \\ A_{21}^{m} & A_{22}^{m} & A_{23}^{m} & A_{24}^{m} \\ A_{31}^{m} & A_{32}^{m} & A_{33}^{m} & A_{34}^{m} \\ A_{41}^{m} & A_{42}^{m} & A_{43}^{m} & A_{44}^{m}\end{array}\right]+\left[\begin{array}{cccc}\left(k_{1}+k_{3}\right) h^{2} I & 0 & 0 & -k_{5} h^{2} I \\ -k_{1} h^{2} I & k_{2} h^{2} I & -k_{4} h^{2} I & 0 \\ 0 & -k_{2} h^{2} I & k_{4} h^{2} I & 0 \\ -k_{3} h^{2} I & 0 & 0 & k_{5} h^{2} I\end{array}\right]$

where $I$ is the $(N-2) \times(N-2)$ identity matrix. The blocks $A_{i j}^{m}$ are $(N-2) \times(N-2)$ penta-diagonal matrices corresponding to the discretization stencil:

$$
\begin{gathered}
{\left[\begin{array}{c}
C W \\
C M \\
C S
\end{array} \quad C E\right.} \\
C M=\frac{h^{2}}{\Delta t}+\left(D_{i j}^{k+\frac{1}{2}, l}+D_{i j}^{k-\frac{1}{2}, l}+D_{i j}^{k, l+\frac{1}{2}}+D_{i j}^{k, l-\frac{1}{2}}\right) \\
C E=-D_{i j}^{k+\frac{1}{2}, l} \\
C W=-D_{i j}^{k-\frac{1}{2}, l} \\
C S=-D_{i j}^{k, l-\frac{1}{2}} \\
C N=-D_{i j}^{k, l+\frac{1}{2}}
\end{gathered}
$$

The linear solvers employed in the modified Picard iteration are the Matlab R2018a implementations of the preconditioned BICGSTAB (see Ref. [2, p. 
24] and also Ref. [21]) and preconditioned restarted GMRES (m) (see Ref. [2, p. 19] and also Ref. [21]). The preconditioners tested are the block diagonal matrices:

$$
\left[\begin{array}{cccc}
\tilde{\Delta_{0}} & 0 & 0 & 0 \\
0 & \tilde{\Delta_{0}} & 0 & 0 \\
0 & 0 & \tilde{\Delta_{0}} & 0 \\
0 & 0 & 0 & \tilde{\Delta_{0}}
\end{array}\right]
$$

where $\tilde{\Delta_{0}}$ is the complete/incomplete Cholesky factorization (symbolized by $\mathrm{CC}=\mathrm{IC}$ ) of the discrete Laplace operator. The incomplete Cholesky factorization preserves the sparsity structure of the original matrix. Details concerning the preconditioners will be given in the next section. In all numerical experiments, the value of the GMRES (m) restart parameter was:

$$
m=35
$$

The stopping criterion for both the linear and non-linear iterations is:

$$
\frac{\left\|r e s_{i}\right\|}{\left\|r e s_{0}\right\|}<10^{-6}
$$

\subsection{The Preconditioning Techniques}

The preconditioning technique used in the present work is described in detail and theoretical analysed in [4]. The present test problem is similar to that employed in [4]. For these reasons, only the main elements of the preconditioning technique can be seen in the next paragraphs. We consider the system of linear equations to be solved in one step of the Picard method:

$$
A Z=B
$$

where

$$
A=\left[\begin{array}{llll}
A_{11}^{m} & A_{12}^{m} & A_{13}^{m} & A_{14}^{m} \\
A_{21}^{m} & A_{22}^{m} & A_{23}^{m} & A_{24}^{m} \\
A_{31}^{m} & A_{32}^{m} & A_{33}^{m} & A_{34}^{m} \\
A_{41}^{m} & A_{42}^{m} & A_{43}^{m} & A_{44}^{m}
\end{array}\right], Z=\left[\begin{array}{l}
Z_{1} \\
Z_{2} \\
Z_{3} \\
Z_{4}
\end{array}\right], B=\left[\begin{array}{l}
b_{1} \\
b_{2} \\
b_{3} \\
b_{4}
\end{array}\right]
$$

The blocks $A_{i j}$ are squared matrix with dimension $(N-2)^{2}$ and blocks $A_{i i}$, $i=\overline{1,4}$ are symmetric and positive definite. The classical method of constructing a preconditioning matrix for the system (2.31) is based on the Choleski type decomposition, complete or incomplete of the diagonal blocks:

$$
A_{i i}=C_{i} C_{i}^{T}+R_{i}, \quad i=\overline{1,4}
$$


(if $R_{i}=0, \quad i=\overline{1,4}$ we refer to complete decomposition, thus we have the case of incomplete decomposition).

In the case of incomplete decomposition, the matrix $R_{i}$ is different from the null matrix in the same way as the matrix $C_{i}$ maintain the sparsity character of the matrix $A_{i i}$. We choose that the preconditioner are the Jacobi blockdiagonal matrix:

$$
\begin{aligned}
M= & {\left[\begin{array}{cccc}
C_{1} & 0 & 0 & 0 \\
0 & C_{2} & 0 & 0 \\
0 & 0 & C_{3} & 0 \\
0 & 0 & 0 & C_{4}
\end{array}\right]\left[\begin{array}{cccc}
C_{1}^{T} & 0 & 0 & 0 \\
0 & C_{2}^{T} & 0 & 0 \\
0 & 0 & C_{3}^{T} & 0 \\
0 & 0 & 0 & C_{4}^{T}
\end{array}\right] } \\
& =\left[\begin{array}{cccc}
C_{1} C_{1}^{T} & 0 & 0 & 0 \\
0 & C_{2} C_{2}^{T} & 0 & 0 \\
0 & 0 & C_{3} C_{3}^{T} & 0 \\
0 & 0 & 0 & C_{4} C_{4}^{T}
\end{array}\right]
\end{aligned}
$$

The following preconditioning methods can be used:

-left preconditioning:

$A Z=B \quad \Longleftrightarrow \hat{A} Z=\hat{B}, \quad$ with $\quad \hat{A}=M^{-1} A, \quad \hat{B}=M^{-1} B ;$

-right preconditioning:

$A Z=B \quad \Longleftrightarrow \hat{A} \hat{Z}=B, \quad$ with $\quad \hat{A}=A M^{-1}, \quad \hat{Z}=M Z ;$

-left-right preconditioning:

$A Z=B \Longleftrightarrow \hat{A} \hat{Z}=\hat{B}, \quad$ with

$$
\begin{gathered}
\hat{A}=\left[\begin{array}{cccc}
C_{1}^{-1} & 0 & 0 & 0 \\
0 & C_{2}^{-1} & 0 & 0 \\
0 & 0 & C_{3}^{-1} & 0 \\
0 & 0 & 0 & C_{4}^{-1}
\end{array}\right] A\left[\begin{array}{cccc}
C_{1}^{-T} & 0 & 0 & 0 \\
0 & C_{2}^{-T} & 0 & 0 \\
0 & 0 & C_{3}^{-T} & 0 \\
0 & 0 & 0 & C_{4}^{-T}
\end{array}\right] \\
\hat{Z}=\left[\begin{array}{cccc}
C_{1}^{T} & 0 & 0 & 0 \\
0 & C_{2}^{T} & 0 & 0 \\
0 & 0 & C_{3}^{T} & 0 \\
0 & 0 & 0 & C_{4}^{T}
\end{array}\right] Z, \hat{B}=\left[\begin{array}{cccc}
C_{1}^{-1} & 0 & 0 & 0 \\
0 & C_{2}^{-1} & 0 & 0 \\
0 & 0 & C_{3}^{-1} & 0 \\
0 & 0 & 0 & C_{4}^{-1}
\end{array}\right] B
\end{gathered}
$$

Jacobi block-type preconditioning techniques give quite good results, but require four complete or incomplete Choleski-type decompositions of diagonal blocks, which becomes computationally expensive in practical applications.

Next we propose a preconditioning matrix that is no longer based on the diagonal blocks $A_{i i}$, but on a single block, $\Delta_{0}$.

$\Delta_{0}$ is the matrix obtained from the discretization, using finite difference schemes, of the equation $-\Delta z+\frac{\partial z}{\partial t}=0$. The matrix $\Delta_{0}$ is symmetric and positive definite (strictly diagonal dominant and the diagonal elements strictly positive). 
For $N=5, \Delta_{0}$ has the following expression:

$$
\Delta_{0}=\left[\begin{array}{ccccccccc}
4 & -1 & 0 & -1 & 0 & 0 & 0 & 0 & 0 \\
-1 & 4 & -1 & 0 & -1 & 0 & 0 & 0 & 0 \\
0 & -1 & 4 & 0 & 0 & -1 & 0 & 0 & 0 \\
-1 & 0 & 0 & 4 & -1 & 0 & -1 & 0 & 0 \\
0 & -1 & 0 & -1 & 4 & -1 & 0 & -1 & 0 \\
0 & 0 & -1 & 0 & -1 & 4 & 0 & 0 & -1 \\
0 & 0 & 0 & -1 & 0 & 0 & 4 & -1 & 0 \\
0 & 0 & 0 & 0 & -1 & 0 & -1 & 4 & -1 \\
0 & 0 & 0 & 0 & 0 & -1 & 0 & -1 & 4
\end{array}\right]
$$

We define

$$
\tilde{\Delta_{0}}=\Delta_{0}+h^{2} I
$$

where $I$ is the $(N-2)^{2}$ identity matrix.

From construction $\tilde{\Delta_{0}}$ is the symmetric and positive definite matrix $\left(\left\langle\tilde{\Delta_{0}} x, x\right\rangle=\right.$ $\left\langle\Delta_{0} x, x\right\rangle_{h}^{2}\|x\|^{2}>0, \quad \forall x \neq 0$ ) and accept a Cholesky decomposition (for $R=0$ is complete, otherwise is incomplete)

$$
\tilde{\Delta_{0}}=C C^{T}+R
$$

The preconditioning matrix used in this work is:

$P=\left[\begin{array}{cccc}\tilde{\Delta_{0}} & 0 & 0 & 0 \\ 0 & \tilde{\Delta_{0}} & 0 & 0 \\ 0 & 0 & \tilde{\Delta_{0}} & 0 \\ 0 & 0 & 0 & \tilde{\Delta_{0}}\end{array}\right]=\left[\begin{array}{cccc}C & 0 & 0 & 0 \\ 0 & C & 0 & 0 \\ 0 & 0 & C & 0 \\ 0 & 0 & 0 & C\end{array}\right]\left[\begin{array}{cccc}C^{T} & 0 & 0 & 0 \\ 0 & C^{T} & 0 & 0 \\ 0 & 0 & C^{T} & 0 \\ 0 & 0 & 0 & C^{T}\end{array}\right]=\Gamma \cdot \Gamma^{T}$

\section{NUMERICAL RESULTS}

In the case of the linear stationary diffusion-reaction problem, the decrease of the convergence rate and the efficiency of the algorithms was observed due to the increase of the diffusion interaction of chemical species, while in the nonlinear case, the diffusion interaction does not significantly influence the convergence speed and efficiency [3].

In the case of the linear stationary convection-diffusion-reaction problem, the decrease in the convection rate leads to the increase of the diffusion interaction, and in the nonlinear case, the increase of the dilution ratio leads to the increase of the convergence rate and efficiency [3].

For the non-stationary linear case, a splitting method was developed for the 
convection-diffusion problem. The advantage of the splitting method is that the algebraic systems to be solved are triangular blocks. The stability of the method is given by the condition that the two triangular block matrices (upper and lower) are positively defined [6,7].

The multicomponent diffusion-reaction problem analyzed in this paper is time dependent (contains an evolving term) and is non-linear (due to the fact that the diffusion coefficients are dependent on concentrations).

The present computations are focused on the following aspects: stability, accuracy and the numerical performances of the algorithms employed. The time step is considered constant and takes values from $\Delta t=0.001$ to $\Delta t=0.01$. The values used for the spatial grid step size $h$ are $h=2^{-5}, 2^{-6}, 2^{-7}$ and $2^{-8}$. The influence of cross-diffusion coupling on the numerical performances of the numerical algorithms is analysed considering the following multicomponent Fick diffusion matrices:

$$
\begin{aligned}
{\left[\begin{array}{cccc}
D_{11} & 0 & 0 & 0 \\
0 & D_{22} & 0 & 0 \\
0 & 0 & D_{33} & 0 \\
0 & 0 & 0 & D_{44}
\end{array}\right] } & {\left[\begin{array}{cccc}
D_{11} & D_{12} & 0 & 0 \\
D_{21} & D_{22} & 0 & 0 \\
0 & 0 & D_{33} & 0 \\
0 & 0 & 0 & D_{44}
\end{array}\right]\left[\begin{array}{cccc}
D_{11} & D_{12} & D_{13} & 0 \\
D_{21} & D_{22} & D_{23} & 0 \\
D_{31} & D_{32} & D_{33} & 0 \\
0 & 0 & 0 & D_{44}
\end{array}\right] } \\
& { }_{(b)} \\
& {\left[\begin{array}{cccc}
D_{11} & D_{12} & D_{13} & D_{14} \\
D_{21} & D_{22} & D_{23} & D_{24} \\
D_{31} & D_{32} & D_{33} & D_{34} \\
D_{41} & D_{42} & D_{43} & D_{44}
\end{array}\right] }
\end{aligned}
$$

For these matrices, the cross-diffusion coupling increases progressively, from no cross-diffusion coupling (case (a)) to all species interaction (case (d)). For brevity, the case (a) will be symbolized by CPL1, case (b) by CPL2, case (c) by CPL3 and case (d) by CPL4. The values of the Stefan-Maxwell diffusion coefficients are presented in the Appendix. The values of the dimensionless reaction rate constants are:

$$
\begin{aligned}
& (1)-k_{1}=1, \quad k_{2}=0.4, \quad k_{3}=0.5, \quad k_{4}=0.2, \quad k_{5}=0.1 \\
& (2)-k_{1}=10, \quad k_{2}=4, \quad k_{3}=5, \quad k_{4}=2, \quad k_{5}=1 \\
& (3)-k_{1}=100, \quad k_{2}=40, \quad k_{3}=50, \quad k_{4}=20, \quad k_{5}=10
\end{aligned}
$$



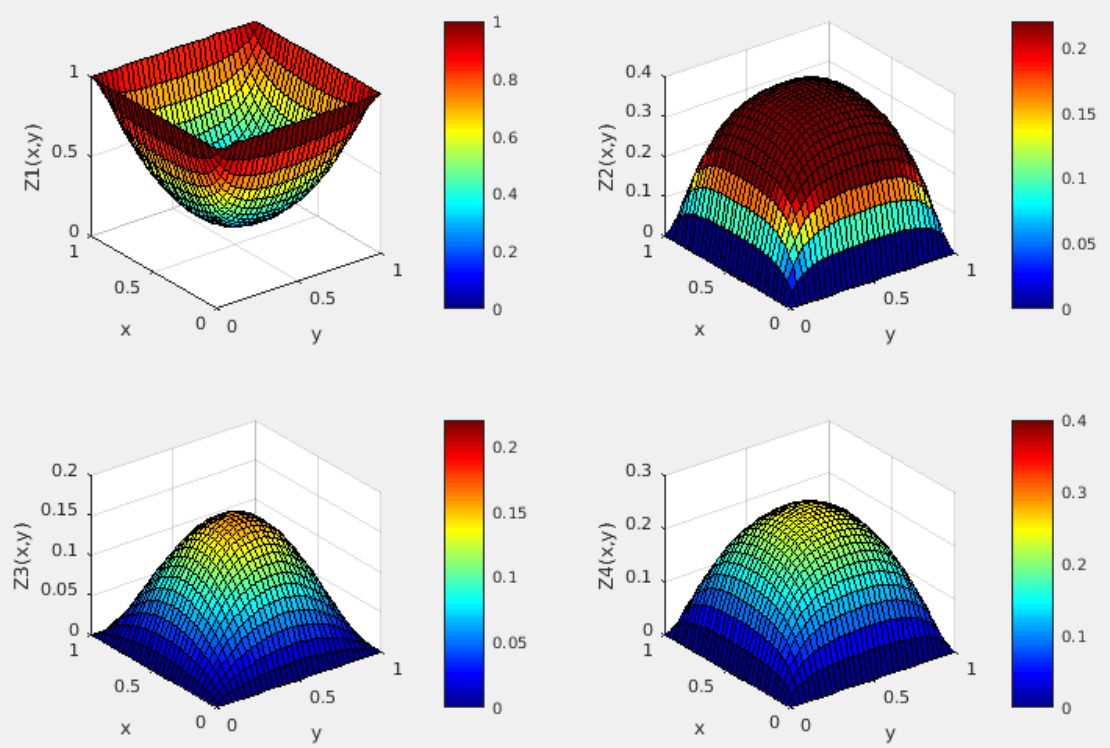

1: Dimensionless concentration profiles calculated for set B1, set (2) of dimensionless reaction rate constants, $Z_{5 b}=1, \mathrm{CPL}=4$, mesh 33x33, GMRES(Non)
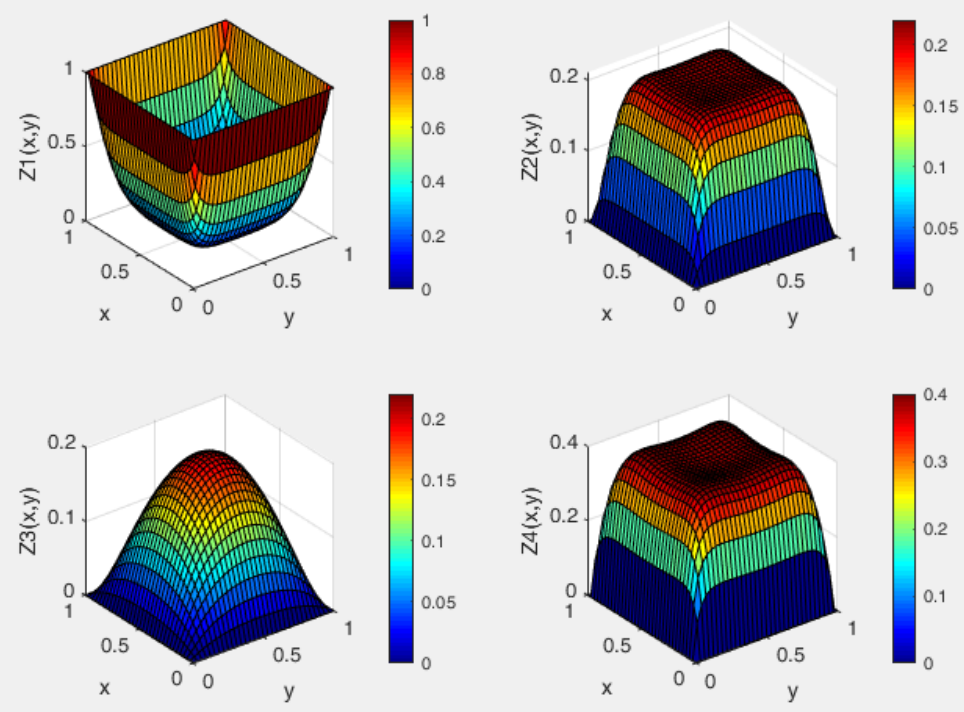

2: Dimensionless concentration profiles calculated for set B2, set (3) of dimensionless reaction rate constants, $Z_{5 b}=1, \mathrm{CPL}=4$, mesh 33x33, GMRES(Non) 
The numerical results for set $B_{1}$ of Stefan-Maxwell diffusion coefficients and set(2) of dimensionless reaction rate constants are plotted in Figure 1. The numerical results for set $B_{2}$ of Stefan-Maxwell diffusion coefficients and set(3) of dimensionless reaction rate constants are depicted in Figure 2. The results introduced in Figure 1-2 were gotten utilizing a constant time step equal to $\Delta t=10^{-3}$ and final time $t=1$.

The numerical results rich a stationary state, at time $t=1$.

All numerical results obtained confirm the stability of the method. For all the combinations of the parameters we obtained a stable solution.

To analyse the spatial convergence rate, we made a convergence analysis using the following sequence of spatial grids: $33 \times 33,65 \times 65,129 \times 129$, and $257 \times 257$. Let $Z^{(m-1)}$ and $Z^{(m)}$ denote the solutions on grid $m 1$ and the next finer grid $m$ with twice as many points in both coordinate directions. The difference between the solutions on the grids $m 1$ and $m$ is given by (in the discrete $p$ norm)

$$
\operatorname{Err}^{(m)}=\left\|Z^{(m)}-Z^{(m-1)}\right\|_{p}
$$

The rate of convergence can be approximated by formula [15]:

$$
R^{(m)} \approx \log _{2}\left(\frac{\operatorname{Err}^{(m-1)}}{\operatorname{Err}(m)}\right)
$$

Tables 1-2 show a selection from the numerical experiments made for the calculation of the spatial convergence rate. The norm used is the discrete Euclidean norm $(p=2)$.

We observe that, for $B_{1}$ and $B_{2}$, and the grid $m=3,4, R(m)$ are approximately equal to 2.3 . The values of $R(\mathrm{~m})$ previously presented show that, as expected, the present numerical solution is second-order accurate in space. 
ON NUMERICAL SOLUTION OF NONLINEAR PARABOLIC MULTICOMPONENT DIFFUSION-REACTION PROBLEMS

Table 1: Convergence rate ; set $B_{1}$ of the Stefan-Maxwell diffusion coefficients

\begin{tabular}{|c|c|c|c|c|c|c|}
\hline \multirow[t]{2}{*}{$\mathrm{m}$} & \multirow[t]{2}{*}{ Mesh } & \multirow[t]{2}{*}{ Case } & \multicolumn{2}{|c|}{$Z_{5 b}=1$} & \multicolumn{2}{|c|}{$Z_{5 b}=10$} \\
\hline & & & $\operatorname{Err}(\mathrm{m})$ & $\mathrm{R}(\mathrm{m})$ & $\operatorname{Err}(m)$ & $\mathrm{R}(\mathrm{m})$ \\
\hline \multirow[t]{4}{*}{1} & $33 \times 33$ & CPL1 & - & - & - & - \\
\hline & & CPL2 & - & - & - & - \\
\hline & & CPL3 & - & - & - & - \\
\hline & & CPL4 & - & - & - & - \\
\hline \multirow[t]{4}{*}{2} & $65 \times 65$ & CPL1 & 0.002 & - & 0.002 & - \\
\hline & & CPL2 & 0.002 & - & 0.002 & - \\
\hline & & CPL3 & 0.0019 & - & 0.002 & - \\
\hline & & CPL4 & 0.0019 & - & 0.002 & - \\
\hline \multirow[t]{4}{*}{3} & $129 \times 129$ & CPL1 & 0.00041 & 2.286304 & 0.00042 & 2.251539 \\
\hline & & CPL2 & 0.00041 & 2.286304 & 0.00042 & 2.251539 \\
\hline & & CPL3 & 0.00041 & 2.212304 & 0.00041 & 2.286304 \\
\hline & & CPL4 & 0.00040 & 2.247928 & 0.00041 & 2.286304 \\
\hline \multirow[t]{4}{*}{4} & $257 \times 257$ & CPL1 & 0.000085 & 2.270089 & 0.000086 & 2.287981 \\
\hline & & CPL2 & 0.000085 & 2.270089 & 0.000085 & 2.304855 \\
\hline & & CPL3 & 0.000083 & 2.304441 & 0.000085 & 2.270089 \\
\hline & & CPL4 & 0.000082 & 2.286304 & 0.000084 & 2.287163 \\
\hline
\end{tabular}

Table 2: Convergence rate ; set $B_{2}$ of the Stefan-Maxwell diffusion coefficients

\begin{tabular}{|c|c|c|c|c|c|c|}
\hline \multirow[t]{2}{*}{$\mathrm{m}$} & \multirow[t]{2}{*}{ Mesh } & \multirow[t]{2}{*}{ Case } & \multicolumn{2}{|c|}{$Z_{5 b}=1$} & \multicolumn{2}{|c|}{$Z_{5 b}=10$} \\
\hline & & & $\operatorname{Err}(m)$ & $\mathrm{R}(\mathrm{m})$ & $\operatorname{Err}(\mathrm{m})$ & $\mathrm{R}(\mathrm{m})$ \\
\hline \multirow[t]{4}{*}{1} & $33 \times 33$ & CPL1 & - & - & - & - \\
\hline & & CPL2 & - & - & - & - \\
\hline & & CPL3 & - & - & - & - \\
\hline & & CPL4 & - & - & - & - \\
\hline \multirow[t]{4}{*}{2} & $65 \times 65$ & CPL1 & 0.0021 & - & 0.0021 & - \\
\hline & & CPL2 & 0.0021 & - & 0.0022 & - \\
\hline & & CPL3 & 0.0022 & - & 0.0022 & - \\
\hline & & CPL4 & 0.0021 & - & 0.0022 & - \\
\hline \multirow[t]{4}{*}{3} & $129 \times 129$ & CPL1 & 0.00044 & 2.254814 & 0.00045 & 2.222392 \\
\hline & & CPL2 & 0.00044 & 2.254814 & 0.00045 & 2.289507 \\
\hline & & CPL3 & 0.00045 & 2.289507 & 0.00045 & 2.289507 \\
\hline & & CPL4 & 0.00044 & 2.254814 & 0.00045 & 2.289507 \\
\hline \multirow[t]{4}{*}{4} & $257 \times 257$ & CPL1 & 0.000090 & 2.289507 & 0.000091 & 2.305987 \\
\hline & & CPL2 & 0.000091 & 2.273565 & 0.000091 & 2.305987 \\
\hline & & CPL3 & 0.000091 & 2.305987 & 0.000090 & 2.321928 \\
\hline & & CPL4 & 0.000091 & 2.273565 & 0.000090 & 2.321928 \\
\hline
\end{tabular}


ON NUMERICAL SOLUTION OF NONLINEAR PARABOLIC

\begin{tabular}{|c|c|c|c|c|c|c|c|c|c|}
\hline \multirow{3}{*}{ Mesh } & \multirow{3}{*}{ Case } & \multicolumn{4}{|c|}{ GMRES } & \multicolumn{4}{|c|}{ BiCGSTAB } \\
\hline & & \multicolumn{2}{|c|}{ Non } & \multicolumn{2}{|c|}{ IC } & \multicolumn{2}{|c|}{ Non } & \multicolumn{2}{|c|}{$\mathrm{IC}$} \\
\hline & & $Z_{5 b}=1$ & $Z_{5 b}=10$ & $Z_{5 b}=1$ & $Z_{5 b}=10$ & $Z_{5 b}=1$ & $Z_{5 b}=10$ & $Z_{5 b}=1$ & $Z_{5 b}=10$ \\
\hline \multirow{4}{*}{$33 \times 33$} & CPL1 & 0.2135 & 0.1323 & 0.2136 & 0.1323 & 0.2134 & 0.1320 & 0.2137 & 0.1322 \\
\hline & CPL2 & 0.1906 & 0.1228 & 0.1906 & 0.1225 & 0.1905 & 0.1227 & 0.1907 & 0.1226 \\
\hline & CPL3 & 0.1686 & 0.1176 & 0.1688 & 0.1177 & 0.1687 & 0.1178 & 0.1685 & 0.1175 \\
\hline & CPL4 & 0.1305 & 0.1013 & 0.1303 & 0.1015 & 0.1308 & 0.1014 & 0.1306 & 0.1015 \\
\hline \multirow{4}{*}{$65 \times 65$} & CPL1 & 0.2237 & 0.1348 & 0.2237 & 0.1349 & 0.2236 & 0.1348 & 0.2235 & 0.1348 \\
\hline & CPL2 & 0.2009 & 0.1251 & 0.2013 & 0.1253 & 0.2011 & 0.1250 & 0.2010 & 0.1250 \\
\hline & CPL3 & 0.1765 & 0.1192 & 0.1764 & 0.1190 & 0.1767 & 0.1192 & 0.1768 & 0.1194 \\
\hline & CPL4 & 0.1324 & 0.1029 & 0.1325 & 0.1029 & 0.1325 & 0.1032 & 0.1323 & 0.1030 \\
\hline \multirow{4}{*}{$129 \times 129$} & CPL1 & 0.2294 & 0.1361 & 0.2291 & 0.1364 & 0.2293 & 0.1362 & 0.2294 & 0.1361 \\
\hline & CPL2 & 0.2108 & 0.1308 & 0.2111 & 0.1307 & 0.2107 & 0.1306 & 0.2107 & 0.1307 \\
\hline & CPL3 & 0.1880 & 0.1273 & 0.1881 & 0.1275 & 0.1883 & 0.1274 & 0.1882 & 0.1275 \\
\hline & CPL4 & 0.1394 & 0.1034 & 0.1394 & 0.1033 & 0.1395 & 0.1034 & 0.1394 & 0.1035 \\
\hline
\end{tabular}

Table 3: Average value of the average reduction factor, $\bar{\rho}$; set $B_{1}$ of the Stefan-Maxwell diffusion coefficients

The next issue analysed in this work is the numerical performances of the preconditioned GMRES and BiCGSTAB as linear solvers in the modified Picard iteration. The average reduction factor, $\bar{\rho}$, and the efficiency, $\tau$, are the quantities used to quantify the numerical performances of the preconditioned GMRES and BiCGSTAB. The average reduction factor is given by

$$
\bar{\rho}=\left(\frac{\left\|r e s_{i}\right\|}{\left\|r e s_{0}\right\|}\right)^{\frac{1}{i}}
$$

where res $_{i}$ is the residual after $\mathrm{i}$ iterations. The average reduction factor is one of the quantities widely used to measure the convergence rate of an algorithm. The relation used to compute the efficiency is,

$$
\tau=\frac{W}{|\ln \bar{\rho}|}
$$

where $W$ (the work) is the number of arithmetic operations per grid point and iteration step. The efficiency can be viewed as an indicator for the computational time and implicitly the computational efficiency. 
ON NUMERICAL SOLUTION OF NONLINEAR PARABOLIC

\begin{tabular}{|c|c|c|c|c|c|c|c|c|c|}
\hline \multirow{3}{*}{ Mesh } & \multirow{3}{*}{ Case } & \multicolumn{4}{|c|}{ GMRES } & \multicolumn{4}{|c|}{ BiCGSTAB } \\
\hline & & \multicolumn{2}{|c|}{ Non } & \multicolumn{2}{|c|}{ IC } & \multicolumn{2}{|c|}{ Non } & \multicolumn{2}{|c|}{ IC } \\
\hline & & $Z_{5 b}=1$ & $Z_{5 b}=10$ & $Z_{5 b}=1$ & $Z_{5 b}=10$ & $Z_{5 b}=1$ & $Z_{5 b}=10$ & $Z_{5 b}=1$ & $Z_{5 b}=10$ \\
\hline \multirow{4}{*}{$33 \times 33$} & CPL1 & 0.2135 & 0.1323 & 0.2136 & 0.1323 & 0.2134 & 0.1320 & 0.2137 & 0.1322 \\
\hline & CPL2 & 0.1906 & 0.1228 & 0.1906 & 0.1225 & 0.1905 & 0.1227 & 0.1907 & 0.1226 \\
\hline & CPL3 & 0.1686 & 0.1176 & 0.1688 & 0.1177 & 0.1687 & 0.1178 & 0.1685 & 0.1175 \\
\hline & CPL4 & 0.1305 & 0.1013 & 0.1303 & 0.1015 & 0.1308 & 0.1014 & 0.1306 & 0.1015 \\
\hline \multirow{4}{*}{$65 \times 65$} & CPL1 & 0.2237 & 0.1348 & 0.2237 & 0.1349 & 0.2236 & 0.1348 & 0.2235 & 0.1348 \\
\hline & CPL2 & 0.2009 & 0.1251 & 0.2013 & 0.1253 & 0.2011 & 0.1250 & 0.2010 & 0.1250 \\
\hline & CPL3 & 0.1765 & 0.1192 & 0.1764 & 0.1190 & 0.1767 & 0.1192 & 0.1768 & 0.1194 \\
\hline & CPL4 & 0.1324 & 0.1029 & 0.1325 & 0.1029 & 0.1325 & 0.1032 & 0.1323 & 0.1030 \\
\hline \multirow{4}{*}{$129 \times 129$} & CPL1 & 0.2294 & 0.1361 & 0.2291 & 0.1364 & 0.2293 & 0.1362 & 0.2294 & 0.1361 \\
\hline & CPL2 & 0.2108 & 0.1308 & 0.2111 & 0.1307 & 0.2107 & 0.1306 & 0.2107 & 0.1307 \\
\hline & CPL3 & 0.1880 & 0.1273 & 0.1881 & 0.1275 & 0.1883 & 0.1274 & 0.1882 & 0.1275 \\
\hline & CPL4 & 0.1394 & 0.1034 & 0.1394 & 0.1033 & 0.1395 & 0.1034 & 0.1394 & 0.1035 \\
\hline
\end{tabular}

Table 4: Average values of the efficiency, $\tau$; set $B_{1}$ of the Stefan-Maxwell diffusion coefficients

Tables 3 and 4 presents a selection from the values computed for the average reduction factor and efficiency. This selection captures the salient features of the process. The results presented in tables 3 and 4 lead to the following statements:

- as expected CPL1,CPL2,CPL3,CPL4 and $Z_{5 b}$, has no influnece on the convergence rate

- the convergence rate for the set $B_{1}$ is higher than that for the set $B_{2}$

- for a given mesh, the impact of the cross-diffusion coupling on the efficiency observes similar guidelines as on account of the average reduction factor

\section{CONCLUSIONS}

In this work we have analysed the numerical solving of transient, non-linear multicomponent diffusion-reaction equations in two space dimensions. The non-linear parabolic equations were discretized in time by the fully implicit method. The central second order accurate finite difference scheme was used to discretize the spatial derivatives. The non-linear systems resulted were solved by the modified Picard-preconditioned conjugated gradient methods. For all the combinations of the parameters, we obtained a stable solution. The values of the convergence rate obtained show that the present numerical solution is second-order accurate in space.

The values of the convergence rate and efficiency of the preconditioned 
ON NUMERICAL SOLUTION OF NONLINEAR PARABOLIC

MULTICOMPONENT DIFFUSION-REACTION PROBLEMS

GMRES and BiCGSTAB algorithms are similar those usually reported in literature for non-linear diffusion-reaction problems.

\section{References}

[1] J. Bandrowski and A. Kubaczka, On the Prediction of Diffusivities in Multicomponent Liquid Systems, Chem. Eng. Sci., 37, (1982), 13091313.

[2] R. Barrett, M. Berry, T. F. Chan, J. Demmel, J. Donato, J. Dongarra, V. Eijkhout, R. Pozo, C. Romine, and H. van der Vorst, Templates for the Solution of Linear Systems: Building Blocks for Iterative Methods, SIAM, Philadelphia, PA, (1994).

[3] Gh. Juncu, A. Nicola, C. Popa, and E. Stroila, Preconditioned Conjugate Gradient and Multi-Grid Methods for Numerical Solution of MultiComponent Mass Transfer Equations. II. Convection - Diffusion Reaction Equations, Numer.Heat Transfer A, 66, (2014), 12971319.

[4] Gh. Juncu, A. Nicola, C. Popa, and E. Stroila, Preconditioned Conjugate Gradient and Multi-Grid Methods for Numerical Solution of MultiComponent Mass Transfer Equations. I. Diffusion Reaction Equations, Numer.Heat Transfer A, 66, (2014), 12681296.

[5] Gh. Juncu, Unsteady Ternary Mass Transfer from a Sphere in Creeping Flow Int. J. Therm. Sci., 44, (2005), 255266.

[6] Gh. Juncu, A. Nicola, C. Popa, and E. Stroila, Numerical solution of the parabolic multicomponent convection diffusion mass transfer equations by a splitting method, Numer.Heat Transfer A, 71, (2017), 72-90.

[7] Gh. Juncu, A. Nicola, C. Popa, Splitting methods for the numerical solution of multi-component mass transfer problems Mathematics and Computers in Simulation, 152, (2018), 1-14.

[8] A. Kumar and S. Mazumder, Coupled Solution of the Species Conservation Equations using Unstructured Finite-Volume Method, Int. J. Numer. Methods Fluids, 64, (2010),409442.

[9] E. Kozeschnik, Multicomponent Diffusion Simulation Based on Finite Elements, Metall.Mater. Trans. A, 30A, (1999), 25752582.

[10] A. Leahy-Dios and A. Firoozabadi, Unified Model for Nonideal Multicomponent Molecular Diffusion Coefficients, AIChE J., 53, (2007), 29322939. 
[11] F. Lehmann and Ph. Ackerer, Comparison of Iterative Methods for Improved Solutions of the Fluid Flow Equation in Partially Saturated Porous Media, Transp. Porous Media,31, (1998), 275292.

[12] E. Leonardi and C. Angeli, Transient Diffusion within Spherical Particles: Numerical Resolution of the MaxwellStefan Formulation, Ind. Eng. Chem. Res., 49, (2010), 56545660.

[13] J. C. Maxwell, On the Dynamic Theory of Gases, Philos. Trans. R Soc., 157, (1867), 4988.

[14] S. Mazumder, Critical Assessment of the Stability and Convergence of the Equations of Multi-Component Diffusion, J. Comput. Phys., 212, (2006), 383392.

[15] R.D. Richtmyer and K.W. Morton, Difference Methods for Initial Value Problems, Wiley, New York, (1967).

[16] J. Stefan, Uber das Gleichgewicht und die Bewegung insbesondere die Diffusion von Gasgemengen, Akad. Wiss. Wien, 63, (1871), 63124.

[17] A. Spille-Kohoff, E. Preuss, and K. Bttcher, Numerical Simulation of Multi-Component Species Transport in Gases at Any Total Number of Components, Int. J. Heat Mass Transfer, 55, (2012), 53735377.

[18] J. M. Stockie, K. Promislow, and B. R. Wetton, A Finite Volume Method for Multicomponent Gas Transport in a Porous Fuel Cell Electrode, Int. J. Numer. Methods Fluids, 41, (2003), 577599.

[19] R. Taylor and R. Krishna, Multicomponent Mass Transfer, Wiley, New York, (1993).

[20] W. III. Wangard, D. S. Dandy, and B. J. Miller, A Numerically Stable Method for Integration of the Multicomponent Species Diffusion Equations, J. Comput. Phys.,174, (2001), 460472.

[21] R. Weiss, Parameter-Free Iterative Linear Solvers, Akademie, Berlin, (1996).

Gheorghe JUNCU,

Catedra Inginerie Chimica,

Politehnica University Bucharest,

Polizu 1, Bucharest 011061, Romania.

Email: juncu@cael.pub.ro 
Constantin POPA,

Department of Mathematics,

Ovidius University of Constanta,

Bdul Mamaia 124, 900527 Constanta, Romania.

Email: cpopa1956@gmail.com

Gheorghe SÂRBU,

Department of Oceanography, Coastal and Marine Engineering,

National Institute for Marine Research and Development "Grigore Antipa",

300 Mamaia Blvd., 900581 Constanta, Romania

Email: ghesarbu@gmail.com

\section{APPENDIX}

The values of the stefan-Maxwell diffusion coefficients used for the numerical experiments of Section 4 are:

-set $B_{1}$ :

$$
\begin{array}{cccc}
\mathrm{Ð}_{12}=0.22, & \mathrm{Ð}_{13}=0.31, & \mathrm{Ð}_{14}=0.25, & \mathrm{Ð}_{15}=1.0 \\
\mathrm{Ð}_{23}=0.35, & \bigoplus_{24}=0.1, & \bigoplus_{25}=1.18 \\
& \bigoplus_{34}=0.43, & \bigoplus_{35}=1.2 \\
& & \bigoplus_{45}=1.3
\end{array}
$$

- set $B_{2}$ :

$$
\begin{aligned}
& \bigoplus_{12}=6.326, \quad \bigoplus_{13}=4.2523, \quad \bigoplus_{14}=0.247, \quad \bigoplus_{15}=1.0 \\
& \bigoplus_{23}=4.978, \quad \bigoplus_{24}=0.189, \quad \bigoplus_{25}=5.178 \\
& \mathrm{D}_{34}=3.325, \quad \mathrm{D}_{35}=1.27 \\
& \mathrm{D}_{45}=0.987
\end{aligned}
$$

